\title{
An Analytical Model for Defect Depth Estimation Using Pulsed Thermography
}

\author{
S.L. Angioni ${ }^{1}$ - F. Ciampa $^{1}$ - F. Pinto ${ }^{1}$ - G. Scarselli ${ }^{2}$ D.P. Almond ${ }^{1} \cdot$ M. Meo ${ }^{1}$ (D)
}

Received: 16 September 2015 / Accepted: 28 January 2016 / Published online: 21 March 2016

(C) The Author(s) 2016. This article is published with open access at Springerlink.com

\begin{abstract}
The use of pulsed thermography as a nondestructive evaluation tool for damage monitoring of composite materials has dramatically increased in the past decade. Typically, optical flashes are used as external heating sources, which may cause poor defect definition especially for thicker materials or multiple delaminations. SMArt thermography is a new alternative to standard pulsed thermography as it overcomes the limitations on the use of external thermal sources. Such a novel technology enables a built-in, fast and in-depth assessment of both surface and internal material defects by embedding shape memory alloy wires in traditional carbon fibre reinforced composite laminates. However, a theoretical model of thermal wave propagation for SMArt thermography, especially in the presence
\end{abstract}

M. Meo

m.meo@bath.ac.uk

S.L. Angioni

s.1.angioni@bath.ac.uk

F. Ciampa

f.ciampa@bath.ac.uk

F. Pinto

f.pinto@bath.ac.uk

G. Scarselli

gennaro.scarselli@unisalento.it

D.P. Almond

d.p.almond@bath.ac.uk

1 Department of Mechanical Engineering, University of Bath, BA2 7AY Bath, UK

2 Department of Engineering for Innovation, Università del Salento, 73100 Lecce, Italia of internal structural defects, is needed to better interpret the observations/data measured during the experiments. The objective of this paper was to develop an analytical model for SMArt thermography to predict the depth of flaws/damage within composite materials based on experimental data. This model can also be used to predict the temperature contrast on the surface of the laminate, accounting for defect depth, size and opening, thermal properties of material and defect filler, thickness of the component, and intensity of the excitation energy. The results showed that the analytical model gives good predictions compared to experimental data. This paper is one of the first pioneering work showing the use thermography as a quantitative non-destructive tool where defect size and depth could be assessed with good accuracy.

Keywords Composite materials · Pulsed thermography · Embedded shape memory alloy wires · Analytical methods · Defect depth $\cdot$ Thermal properties

- Highlights

- An analytical model for pulsed thermography is presented.

- A methodology is proposed to estimate defect depth in thermography.

- A quantitative method is demonstrated and limitations discussed.

\section{Introduction}

Flash thermography is the most commonly used thermographic non-destructive evaluation (TNDE) technique 
(e. g. [1, 2]). Flash thermograhy is an active TNDE approach [3], which consists in using a number of high intensity flash lamps to heat the surface of the part under inspection. The result is an instantaneous rise in temperature of the surface followed by a cooling as the heat applied to the surface is conducted into the part. The cooling of the surface is monitored by an infrared (IR) camera and the video images are stored in a personal computer (PC) for viewing at the end of the test.

Detection of delamination defects between plies is of the greatest importance for composite laminates, as they can significantly reduce the strength and performance of structures under compressive in-plane loading, eventually giving rise after bucking to global plate instability [4-6].

Adopting flash thermograhy defect sizing from the thermal images has been performed employing both analytical, experimental and numerical techniques by [7-11]. Especially the quantitative prediction of defect depth using flash thermography is an important on-going research topic. The methods reported in literature for this purpose can be distinguished in empirical and experimental. The main techniques were reviewed and contrasted by [12, 13], who used samples with flat-bottom holes as simulated defects in their experiments. Experimental studies [14] have showed that delaminations with a ratio of diameter to depth of the delamination less than 0.5 cannot be detected using thermography.

An analytical model for flash thermography has been developed in [15] which has been found to provide good results when compared against both experimental and finite difference simulations [16]. This model is based on the impulse heating transient response of an adiabatically isolated layer [17, 18] where the thermal response function of the layer is given by the thermal wave interference expression obtained in [19].

Flash thermography presents a series of complications caused by the use of external lamps.

- The positioning of the $\operatorname{lamp}(\mathrm{s})$ from the part under inspection can have a significant impact on test results.

- The heating from a single lamp will be non-uniform, peaking at the center. A more uniform field of heating can be produced by two or more lamps imposing potentially a substantial increase in the cost of the test equipment.

- Lamp filaments and tubes can continue to emit infrared radiation (lamp after glow) for a significant amount of time (seconds) after the lamp has been switched off which may be detected by the IR camera as it is reflected from the test piece or other surfaces into the camera lens. The result can be the generationof an unwanted IR fog that can obscure required IR images of the surface of the part under inspection. The effect is extremely important for parts whose defect response times are very short $(<1 \mathrm{~s})$ as on this time scale after glow will be very significant.

- The optical characteristics of the surface of the material or its coating heavily impact the results of the inspection. Many materials for example are translucent, i. e. light is absorbed in them over a range of depths (and consequently the heat source obtained by flash excitation is not confined to the surface; it is distributed over a range of depths), but their optical absorption coefficients are not readily available.

In this paper a novel approach to pulsed thermography, which does not require external lamps, but is instead based on SMArt composites, is considered. A SMArt composite is a new kind of smart multifunctional material obtained by embedding shape memory alloy (SMA) wires within traditional carbon fibre-reinforced composite (CFRP) composites [20].

Smart materials are materials designed to adapt to the environment in a controlled fashion following the modification of one or more of their properties by external stimuli [21].

Multifunctional materials lead to optimal system performance, by combining different functions into a single material, otherwise not possible through independent subsystem optimization [22].

Specifically, the embodiment of SMA within the lay-up of CFRP laminates has been widely studied as a valid manufacturing procedure to enhance the impact resistance of traditional composite structures due to their unique physical properties such as shape memory effect and superelasticity [23]. The electrical variation of embedded SMA wires to the strain distribution within hybrid glass-fiber reinforced plastic (GFRP) laminates has been correlated by [24], while a hybrid CFRP/SMA composite with damages suppression function that is enabled by activating the martensite-austenite transformation was manufactured by [25].

However, to this date, only few works have investigated the possibility of exploiting the presence of an internal hybrid grid to enable the laminate to have multiple additional features, hence resulting in a real multifunctional system characterized by specific properties that go further than the traditional load-bearing functionality. Based on these premises, a SMA based multifunctional composite (named SMArt) was developed exploiting their intrinsic thermoelectric properties for applications such as sensing, thermography and de-icing. 
In particular SMArt thermography is a form of material enabled thermography [26] where the embedded SMA wires are used as heat sources to generate in situ power resistive heating (ohmic heating or joule heating) that can be used to perform pulsed thermography. Although good results in term of damage detection have been already obtained with this new technique, there is still the need for a rigorous theoretical frame in which this phenomenon must be included. Such a model should not be limited to the inclusion of SMA, but should be extendable to any kind of conductive wire embedded into the traditional lay-up of a composite laminate.

In this paper an analytical model for pulsed thermography using SMArt composites will be developed specifically for in-plane, delamination-like defects. This model can be used to predict the temperature contrast on the surface of the laminate, accounting for

1. defect depth, size and opening;

2. thermal properties of material and defect filler;

3. thickness of the component;

4. depth of the SMA wire and intensity of the excitation energy.

Moreover, using this model an analytical expression for evaluating the defect depth will be obtained.

Finally, the results of the analytical model and defect depth evaluation are contrasted against experimental results.

"Methods" describes the theory behind the analytical model and its application to evaluating analytically the defect depth, while "Results" compares the model against some experimental data. "Conclusions" provides some conclusions from this work and summarizes the major findings.

\section{Methods}

Embedding an SMA wire at a depth $h$ in a composite laminated plate of thickness $L$ and conducting a impulse of resistive heating through this wire, it is possible to identify the presence of a defect using infrared imaging of the variations of the temperature contrast on the surface of the composite laminate. Here we are assuming an inplane, delamination-like circular defect of diameter $D$ at a depth $d$ from the surface of the material, as described in Fig. 1.

No assumptions are made for the laminated fiberreinforced composite in terms of number of layers and direction of the reinforcement fibers in each layer, as in the following (see "Derivation of an analytical model") only homogenized equivalent thermal properties are being considered.

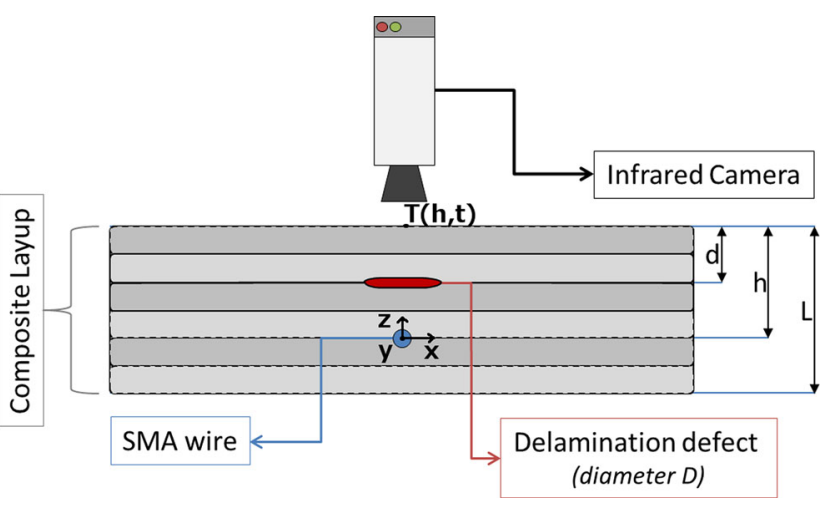

Fig. 1 Identification of an in-plane, delamination-like, circular defect in a composite laminate through pulsed thermography using an SMA wire as heat source. Note that an SMA wire (in blue) is positioned at a depth $h$ from the surface of the composite layup, while a delaminationlike circular defect (in red), of diameter $D$, is positioned at a depth $d$ from the surface. The Cartesian coordinate axes $(x, y, z)$ are oriented such that $x, y$ are in-plane coordinates, with the $y$-axis running along the SMA wire, and $z$ is the transverse coordinate. $T(h, t)$ is the temperature rise on the surface of the material at time $t$

An analytical model for pulsed thermography using an SMA wire as heat source will be derived in the "Derivation of an analytical model". Then "Evaluation of Defect Depth" will explore the possibility of using this model to obtain an approximate expression for evaluating analytically the defect depth.

\section{Derivation of an analytical model}

Consider the laminated fiber-reinforced composite plate shown in Fig. 1, in which the origin of the coordinate system is placed at the center of the SMA wire, which will be assumed to all effects and purposes to act as a line source, and the components of the Cartesian coordinates are $(x, y, z)$ where $x, y$ are in-plane coordinates, and $z$ is the transverse coordinate. Furthermore, it is assumed that the SMA wire runs along the $y$-axis.

The three-dimensional inhomogeneous linear problem of finding the temperature field $T(\boldsymbol{r}, t)$, which results from the transient heat conduction within the composite laminate due to an internal heat source $g(\boldsymbol{r}, t)$, is given in equation (2.1).

$$
\begin{aligned}
\frac{1}{\alpha} \frac{\partial T(\boldsymbol{r}, t)}{\partial t}-\nabla^{2} T(\boldsymbol{r}, t) & =\frac{g(\boldsymbol{r}, t)}{k} & & \Omega \times(0, \infty), t>0 \\
T(\boldsymbol{r}, t) & =0 & & t_{0}=0 \\
T(\boldsymbol{r}, t) & =0 & & S \times(0, \infty), t>0
\end{aligned}
$$

where $\boldsymbol{r}=x \hat{i}+y \hat{j}+z \hat{n}, \Omega \subset \mathbb{R}^{2}$ is the domain of influence, $S$ is the boundary of the composite laminate and 
$\nabla^{2}=\left(\frac{\partial^{2}}{\partial x^{2}}\right) \hat{i}+\left(\frac{\partial^{2}}{\partial y^{2}}\right) \hat{j}+\left(\frac{\partial^{2}}{\partial z^{2}}\right) \hat{n}$ is the Laplacian operator. In equation (2.1) $k$ represents the thermal conductivity of the composite material $\left(\mathrm{W} \mathrm{m}^{-1} \mathrm{~K}^{-1}\right.$ ), whilst $\alpha=\frac{k}{\rho c}$ is the thermal diffusivity of the composite material $\left(\mathrm{m}^{-2}\right)$ with $\rho$ and $c$ the density $\left(\mathrm{kg} \mathrm{m}^{-3}\right)$ and the specific heat capacity ( $\mathrm{W} \mathrm{kg}^{-1} \mathrm{~K}^{-1}$ ) of the composite material, respectively.

The thermal properties in equation (2.1) are the homogenized equivalent thermal properties of the laminated fiberreinforced composite. (In the case of unidirectionallyreinforced fiber composites, the physical properties are anisotropic, but it is common to assume these materials to be transversely isotropic. The anisotropy in thermal conductivity will be accounted for, in the following, using a specific parameter, the thermal diffusivity anisotropy, see equation (2.5) on page 13, whose effects have been studied by one of the authors in [15].)

After resistive heating, if a uniform impulse of thermal energy is released instantaneously by the SMA wire at time $t=0$ and $x=z=0$, but along the entire $y$-axis in a circular cylindrical system of coordinates in the amount (per unit length) $Q_{0} \mathrm{Jm}^{-1}$, then it is a well known result (e. g. [27]) that the temperature is independent of $y$ and the corresponding fundamental solution is given as

$T(r, t)=\frac{Q_{0}}{4 \pi k t} e^{-\frac{r^{2}}{4 \alpha t}}$

where $r=\sqrt{x^{2}+z^{2}}$ and $T(r, t)$ is the temperature rise at a distance $r$ from the SMA wire at a time $t$ after the impulse of heat energy has been released. We are interested in the cross-sectional propagation of the transient thermal wave (i. e. along the $\mathrm{z}$-axis), so the corresponding one-dimensional (1D) field solution of problem equation (2.1) is independent of $x$ and $y$.

When there is no defect in the component being inspected the heat released by the SMA wire will propagate straight through the laminated fiber-reinforced composite plate without impediments causing over time a temperature rise on the surface at a distance $h$ from the wire. The temperature rise $T_{n d}(h, t)$ on the surface of the non-defective material will consist of two contributions.

1. The first contribution $T_{n d}^{F}(h, t)$ is constituted by the forward wave, which reaches directly the surface, and all its subsequent reflections at the back face of the laminate of thickness $L$.

2. The second one $T_{n d}^{R}(h, t)$ instead is composed by the reverse wave, which reaches the surface only after being reflected at the back face of the laminate, and all its subsequent reflections by the same back face.
Note that in theory there are an infinite number of reverberations, but in practice a summation over the first six terms is adequate.

The 1D analytical model of the temperature rise (or background temperature) on the surface of the non-defective material $T_{n d}(h, t)$ is then given by equation (2.3).

$$
\begin{aligned}
T_{n d}(h, t) & =T_{n d}^{F}(h, t)+T_{n d}^{R}(h, t) \\
& =\frac{Q_{0}}{4 \pi k t}\left[\sum_{m=0}^{\infty} e^{-\frac{(2 m L+h)^{2}}{4 \alpha t}}+\sum_{m=0}^{\infty} e^{-\frac{(2(m+1) L-h)^{2}}{4 \alpha t}}\right]
\end{aligned}
$$

Note that the in equation (2.3) the magnitude of the heating produced at the surface varies inversely with the thermal conductivity $k$ of the composite material being impulse heated and that this heat decays with time $t$.

As a delamination-like defect lying beneath the surface in a plane parallel to the surface is being considered (see Fig. 1), then both the magnitude and the time dependence of the temperature rise on the laminate surface, $T(h, t)$, will be altered by the defect, as the conduction of the heat released by the SMA wire to the surface will be reduced or blocked by the defect causing a slower temperature rise than indicated by equation (2.3). This results in the area of the surface over a defect appearing cooler in the IR video images collected during the test, enabling the detection of the defect.

As a first approximation, a region containing a delamination-like defect can be treated as a layer of thickness $d$, the depth of the defect, below the surface.

When a defect is present in the component under inspection, a part $\Gamma$ of the heat, generated by the the SMA wire and being conducted towards the surface will be blocked by the defect, where $\Gamma$ is the effective thermal reflectivity of the defect, assumed $100 \%$ for a wide open defect. This applies to both direct and reverse waves and all their reflections. Then only a fraction $(1-\Gamma)$ of the heat will therefore be able to reach the surface. Another fraction $\Gamma$ of this heat reverberates at the surface after being reflected by the defect having a round-trip path of length $2 d$, with following terms having round-trip path lengths of $4 d, 6 d$, etc.

The 1D analytical model of the temperature rise $T_{d}(h, t)$ on the surface of the defective material is then given by equation (2.4).

$$
\begin{aligned}
T_{d}(h, t) & =T_{d}^{F}(h, t)+T_{d}^{R}(h, t) \\
& =\frac{Q_{0}}{4 \pi k t}\left[\sum_{m=0}^{\infty}(1-\Gamma)^{2 m+1} \sum_{n=0}^{\infty} \Gamma^{n} e^{-\frac{(2 m L+h+2 n d)^{2}}{4 \alpha t}}\right. \\
& \left.+\sum_{m=0}^{\infty}(1-\Gamma)^{2 m+1} \sum_{n=0}^{\infty} \Gamma^{n} e^{-\frac{(2(m+1) L-h+2 n d)^{2}}{4 \alpha t}}\right]
\end{aligned}
$$


Real defects are not infinite in size, but are finite in their lateral dimensions, so heat flowing in their vicinity cannot be assumed to be a $1 \mathrm{D}$ phenomenon. As a circular defect of diameter $D$ has been assumed, then the thermal lateral diffusion around the defect will also contribute to the defective temperature rise over time on the surface of the plate [15]. The physical assumption here is that the lateral diffusion of heat from the edge of the defect to the center will cause an increase with time in the temperature on the surface of the laminate over the center of a defect. For a circular defect of diameter $D$, the diffusion distance is $D / 2$.

Accounting for lateral diffusion, then the temperature rise on the surface of the defective material (equation (2.4)) can be rewritten as

$T_{d_{1}}(h, t)=T_{d}(h, t)+\left(T_{n d}(h, t)-T_{d}(h, t)\right) e^{-\frac{D^{2}}{16 \alpha A t}}$

where $A$ is the thermal diffusivity anisotropy of the composite material. This type of anisotropy assumes a prominent importance on the temperature contrast caused by defects for materials that are thermally anisotropic, such as composites, for which in-plane thermal conductivity typically exceeds through-the-thickness conductivity because of the layered structure of composites. For this reason, the inplane thermal diffusivity of composites is larger by a factor of $\approx$ 3-5 than the through-the-thickness thermal diffusivity. For thermally anisotropic materials the diffusivity should include the anisotropy factor $A$ in the exponential term, while for the thermally isotropic ones $A=1$ in equation (2.5).

The exponential term in equation (2.5), which multiplies the 1D terms, accounts for the physics of the diffusion of heat from the edge of the circular defect to the center, a distance $D / 2$ away. In practice, (equation (2.5)) can be used to provide the temperature rise at any distance from the edge of the defect. This fact will be used in "Results".

The temperature contrast $T_{c}(h, t)$ on the surface of the material is given by equation (2.6)

$$
\begin{aligned}
T_{c}(h, t) & =T_{d_{1}}(h, t)-T_{n d}(h, t) \\
& =\left(T_{d}(h, t)-T_{n d}(h, t)\right)\left(1-e^{-\frac{D^{2}}{16 \alpha A t}}\right)
\end{aligned}
$$

The temperature contrast at the surface over a defective region is negative, it decreases with time until it reaches a minimum, after which it increases tending to zero. A thermal contrast image of the defect will be obtained, only if
1. the magnitude of the minimum temperature contrast is significantly above the noise level of the IR camera (typically $0.02 \mathrm{~K}$ ), and

2. the minimum contrast time is long enough to be recorded by the camera (typical frame rate $60 \mathrm{~Hz}$ ).

Examples of thermal images, along with a further discussion, will be presented in "Results".

Effects of defect opening must also be accounted for, as real defects have a finite opening that may range from less than a $\mu \mathrm{m}$ to several $m \mathrm{~m}$. The thermal barriers presented by such defects are strongly dependent on defect opening and the thermal properties of the host material. Defects are usually treated as thermal contact resistances, $R=l_{d} / k_{d}$, where $l_{d}$ is the defect opening and $k_{d}$ is the thermal conductivity of the material filling the defect (usually it is assumed that defects are air filled).

In previous work [28] on thermal wave interference an expression was obtained for the thermal reflectivity of a defect represented as a thermal contact resistance.

$\Gamma=\frac{R k \sigma}{2+R k \sigma}$

In expression (equation (2.7)) $k$ is the thermal conductivity of the host material and $\sigma$ is the thermal wave number $(1+i) / \mu(\mu$ is the thermal diffusion length). The quantity $2 \sqrt{\alpha t}$ is equivalent to $\mu$ for transient thermal phenomena, where $\alpha$ is the thermal diffusivity of the host material. Making use of this in equation (2.7) leads to expression (equation (2.8)).

$\Gamma=\frac{R \zeta}{2 \sqrt{t}+R \zeta}$

where $\zeta=\sqrt{k \rho c}$ is the thermal effusivity of the composite laminate $\left(\mathrm{Ws}^{1 / 2} /=\mathrm{K}=\mathrm{m}^{2}\right)$.

Expression (equation (2.8)) shows an effective thermal reflectivity of a defect that is a function of its thermal contact resistance $R$ and the thermal properties $k, \rho, c$, of the host material. The results of using this expression have been found to be in good agreement with numerical modeling studies of the effects of defect opening on temperature contrast [10]. Expression (equation (2.8)) is used in (equations (2.4) and (2.5)) to compute the temperature contrast of defects of specified openings.

The temperature contrast can change dramatically depending on the defect opening, so this is a very important parameter whose effects will be analyzed in "Results". 


\section{Evaluation of Defect Depth}

The possibility of obtaining from the model derived in "Derivation of an analytical model" an approximate expression for evaluating analytically the defect depth will be explored in the present subsection.

Considering in equation (2.3) only the first term in the summations in $m$, i. e. $m=0$, and in equation (2.4) only the first term in the summations in $m$ and two terms in the summations in $n$, i. e. $m=0$ and $n=0,1$, then the temperature contrast $T_{c}(h, t)$ can be rewritten as

$$
\begin{aligned}
T_{c}(h, t) & =\left(T_{d}(t)-T_{n d}(t)\right) \\
& =\frac{Q_{0}}{4 \pi k t}\left(\Gamma e^{-\frac{(h+2 d)^{2}}{4 \alpha t}}+\Gamma e^{-\frac{(2 L-h+2 d)^{2}}{4 \alpha t}}\right. \\
& -\Gamma e^{-\frac{h^{2}}{4 \alpha t}}-\Gamma e^{-\frac{(2 L-h)^{2}}{4 \alpha t}} \\
& \left.-\Gamma^{2} e^{-\frac{(h+2 d)^{2}}{4 \alpha t}}-\Gamma^{2} e^{-\frac{(2 L-h+2 d)^{2}}{4 \alpha t}}\right)\left(1-e^{-\frac{D^{2}}{16 \alpha A t}}\right)
\end{aligned}
$$

Neglecting the second and the sixth terms on the righthand side of equation (2.9), an approximate expression for the temperature contrast is given by

$$
\begin{aligned}
T_{c}(h, t) & =-\frac{Q_{0}}{4 \pi k t} \Gamma\left(e^{-\frac{h^{2}}{4 \alpha t}}+e^{-\frac{(2 L-h)^{2}}{4 \alpha t}}\right. \\
& \left.+\Gamma e^{-\frac{(h+2 d)^{2}}{4 \alpha t}}-e^{-\frac{(h+2 d)^{2}}{4 \alpha t}}\right)\left(1-e^{-\frac{D^{2}}{16 \alpha A t}}\right)
\end{aligned}
$$

As discussed in "Derivation of an analytical model", the temperature contrast on the surface of the laminate over a defective area reaches a minimum $T_{c}=T_{c_{\min }}$ at a certain time $t=t_{\min }$ after the pulsed heat excitation has been released by the SMA wire.

Assuming known material and defect thermal properties, geometry of the sample and defect diameter and opening, and substituting $\Gamma$ with the expression in equation (2.8) and the values $T_{c}=T_{c_{\min }}$ and $t=t_{\min }$ derived from the experimental results in equation (2.10), then the following quadratic equation in the defect depth $d$, the only remaining unknown, is obtained

$d^{2}+h d+\left(\frac{h}{2}-q_{9}\right)\left(\frac{h}{2}+q_{9}\right)=T_{c_{\text {min }}}$

with solutions

$$
d_{1}=q_{9}-\frac{h}{2} d_{2}=-\frac{h}{2}-q_{9}
$$

where

$$
\begin{array}{ll}
q_{1}=\left(e^{\frac{D^{2}}{\alpha A t_{\text {min }}}}\right)^{\frac{1}{16}} & q_{2}=e^{\frac{L^{2}}{\alpha t_{\text {min }}}} \\
q_{3}=\left(e^{\frac{h^{2}}{\alpha t_{\text {min }}}}\right)^{\frac{1}{4}} & q_{4}=e^{\frac{L h}{\alpha t_{\text {min }}}} \\
q_{5}=2 Q_{0} R \sqrt{t_{\text {min }}} \zeta q_{1} q_{2} & q_{6}=2 Q_{0} R \sqrt{t_{\min }} \zeta q_{2}
\end{array}
$$

Fig. 2 Comparison between the infrared images for the experimental data for the $30 \mathrm{~ms}$ heating period at different time instants after the impulsive heating excitation. The green rectangle represents a user selectable region of interest used to rescale the digital levels of the visualized thermal images. The boundaries of the plate in Fig. 2(a)-(d) are sketched in red, the SMA wire is located in the center of the hotter region, the unit of the $\mathrm{x}$ and $\mathrm{y}$ axes is pixels and the unit of the colorbars is digital levels

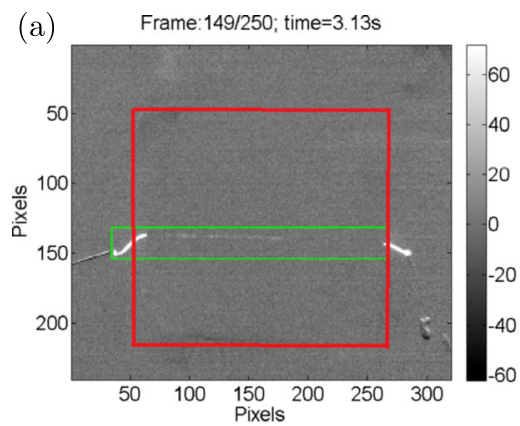

$0.02 \mathrm{~s}$ after the pulsed excitation.

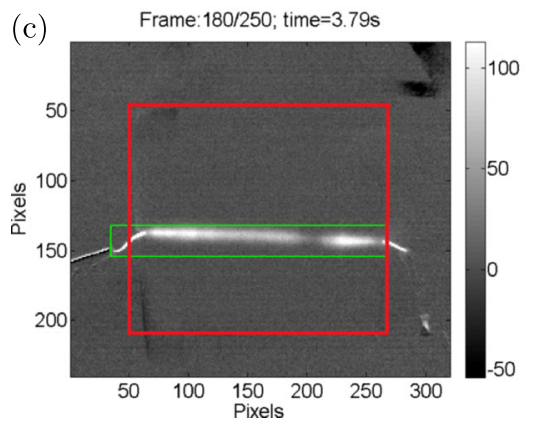

0.64 s after the pulsed excitation.

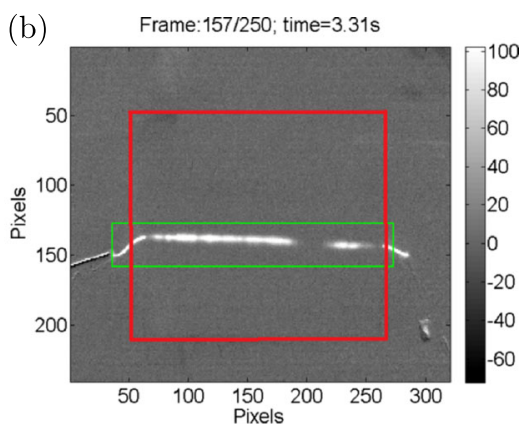

$0.18 \mathrm{~s}$ after the pulsed excitation.



0.94 s after the pulsed excitation. 


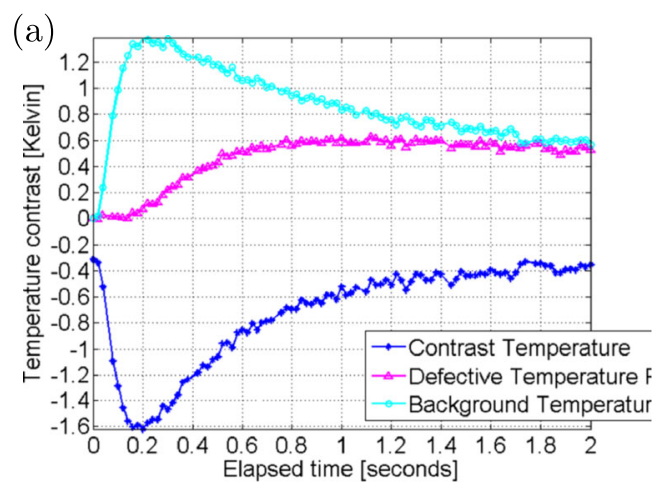

(b)

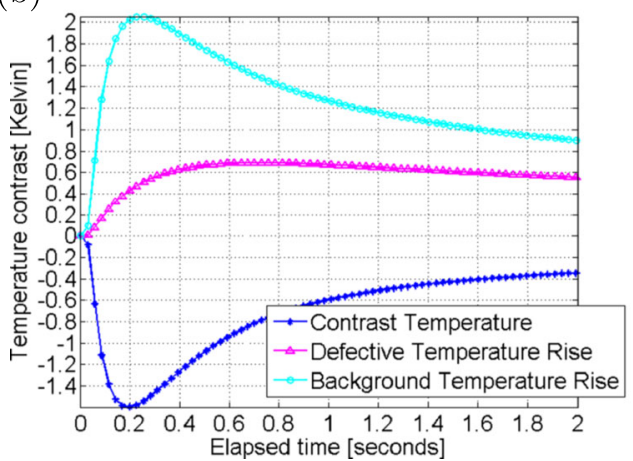

Fig. 3 Results from the analytical model (equation (2.6)) compared against the experimental ones for the $30 \mathrm{~ms}$ heating period

and

$$
\begin{aligned}
q_{7} & =Q_{0} R^{2} \zeta^{2} q_{1} q_{2}-Q_{0} R^{2} \zeta^{2} q_{4} \\
& -2 Q_{0} R \sqrt{t_{\min }} \zeta q_{2}-2 Q_{0} R \sqrt{t_{\min }} \zeta q_{4}-Q_{0} R^{2} \zeta^{2} q_{2} \\
& +Q_{0} R^{2} \zeta^{2} q_{1} q_{4}+2 Q_{0} R \sqrt{t_{\min }} \zeta q_{1} q_{2} \\
& +2 Q_{0} R \sqrt{t_{\min }} \zeta q_{1} q_{4}+16 \pi T_{c \min } k\left(t_{\min }\right)^{2} q_{1} q_{2} q_{3} \\
& +4 \pi R^{2} T_{c \min } k t_{\min } \zeta^{2} q_{1} q_{2} q_{3}+16 \pi R T_{c_{\min }} k\left(t_{\min }\right)^{(3 / 2)} \zeta q_{1} q_{2} q_{3} \\
q_{8} & =q_{6}-q_{5} \\
q_{9} & =\frac{1}{2}\left(h^{2}-4 \alpha t_{\min } \log \left(-\frac{q_{7}}{q_{8}}\right)\right)^{\frac{1}{2}}
\end{aligned}
$$

Only the positive solution $d_{1}$ in equation (2.12) makes physical sense, and can be used to evaluate analytically the defect depth, assumed known all other parameters.

\section{Results}

In this section the results of the analytical model and the approximate expression for evaluating analytically the defect depth, which were presented in "Methods", are compared against experimental data. The analytical model was implemented in MATLAB [29], which is a numerical computing scripting language notably suitable for matrix manipulations.

The experimental results are for a four-ply laminated composite plate with orientations $\left[0^{\circ} 90^{\circ} 90^{\circ} 0^{\circ}\right]$ obtained from a composite $T 700 / M 21$ unidirectional prepreg with fiber volume fraction $\approx 57-59 \%$. Each lamina has a thickness of $\approx 0.1375 \mathrm{~mm}$. The dimensions of the plate were $10 \times$ $6 \times 0.55 \mathrm{~mm}^{3}$ (width $\times$ height $\times$ thickness). A NiTi SMA wire with a diameter of $350 \mu \mathrm{m}$ was positioned between the 3rd and the 4th plies, while the presence of a defective area was modeled using a $11 \mathrm{~cm}^{2}$ Teflon patch of $\approx 0.05 \mathrm{~mm}$ thickness. The inclusion of Teflon inserts is a well-known technique for introducing artificial in-plane delaminations in a laminate composite structure [20, 26, 30].

A pulse of electric potential difference $\Delta V$ was applied at the ends of the SMA wire in order to induce resistive heating in the component under test for a short heating period. The effects of the heating were then captured by the thermal camera in a thermographic system. The thermal camera was an InSb electrically cooled infrared camera with a noise-equivalent temperature difference of $\approx 18-25 \mathrm{mK}$ and a resolution of $320 \times 240$ pixels (width $\times$ height). The camera was used at a frame rate of $50 \mathrm{~Hz}$.

The heating period was varied in the experiments between $10-50 \mathrm{~ms}$ in steps of $10 \mathrm{~ms}$ in order to characterize the impulsive nature of the analytical model. A $\Delta V$ of $2 \mathrm{~V}$ was applied in all tests. An electrical resistance of $2.5 \Omega$ was measured in the SMA wire. All tests were conducted at ambient temperature $\left(\approx 25^{\circ}\right)$.

Figure 2 shows a comparison between the infrared images of the experimental data for the $30 \mathrm{~ms}$ heating period at different time instants after the impulsive heating excitation (which in this case occurred at $\mathrm{t}=3.1 \mathrm{~s}$ ).

The following signal processing techniques were adopted in order to visualize the defect in the thermal images.

1. Background subtraction was applied to the images in Fig. 2 between the pre-excitation and post-excitation thermal images.

Table 1 Thermal properties of CFRP used in the analytical model

\begin{tabular}{llll}
\hline$k\left[\mathrm{Wm}^{-1} \mathrm{~K}^{-1}\right]$ & $c\left[\mathrm{~J} \mathrm{~kg}^{-1} \mathrm{~K}^{-1}\right]$ & $\rho\left[\mathrm{kg} \mathrm{m}^{-1} \mathrm{~K}^{-1}\right]$ & $\alpha 10^{-7}\left[\mathrm{~ms}^{-2}\right]$ \\
\hline 0.50 & 1200 & 1700 & 5.88 \\
\hline
\end{tabular}


Table 2 Results predicted by the analytical model (M) (equation (2.6)) and its approximate expression (AM) (equation (2.10)) for the minimum contrast time $t_{\min }$ compared against the experimental ones for $10 \mathrm{~ms}, 20 \mathrm{~ms}, 30 \mathrm{~ms}, 40 \mathrm{~ms}$ and $50 \mathrm{~s}$ heating periods

\begin{tabular}{lllllllr}
\hline & $\mathrm{M}$ & AM & $10 \mathrm{~ms}$ & $20 \mathrm{~ms}$ & $30 \mathrm{~ms}$ & $40 \mathrm{~ms}$ & $50 \mathrm{~ms}$ \\
\hline$t_{\min }[\mathrm{xS}]$ & 0.19 & 0.20 & 0.20 & 0.24 & 0.20 & 0.18 \\
\hline
\end{tabular}

2. Thermal images were smoothed locally using simple moving averaging.

3. Moreover, the digital levels of the visualized thermal images were also rescaled based on a user selectable region of interest $(\mathrm{ROI})$, which is represented by a green rectangle in the Fig. 2(a)-(d).

Figure 2(a) refers to the thermal image at a time immediately after the pulsed heat excitation, while (Fig. 2(b)) is for a time $\mathrm{t}=0.18 \mathrm{~s}$ after the pulsed excitation where the temperature contrast reaches its minimum value. The presence of the patch is clearly visible in Fig. 2(b) as a break in the temperature rise in the thermal image. Figure 2(c) and (d) show how at times much later there is a temperature rise also on the surface area over the patch caused by heat diffusion both through and around the defective area.

Figure 3(a) shows the results for the experimental data for the 30ms heating period. All graphs in Fig. 3(a) are plotted starting from when the SMA wire starts heating, after $\Delta V$ has been applied, and were smoothed locally using simple moving averaging. The non-defective temperature rise is being measured on the surface of the plate in a small nondefective area directly on top of the SMA wire, so that it is uniform as much as possible. After the initial rise the nondefective temperature decreases because of the effects of thermal convection towards the air surrounding the sample and thermal conduction in-plane to the SMA wire. The temperature is given in Kelvin. The non-defective temperature increases by $\approx 1.50$ Kelvin. The defective temperature rise is measured on the surface of the plate in a small defective area directly on top of the SMA wire, so that it is uniform as much as possible. The graph of the defective temperature rise takes into account non only the effects of thermal diffusion through the defect, but also the effects of lateral thermal diffusion around the defect. The defective temperature rise is of $\approx 0.60$ Kelvin. The temperature contrast in Fig. 3(a) is negative reaching a minimum at 0.20 s. The temperature contrast reduction is of $\approx 1.60$ Kelvin.

Figure 3(b) shows the results from the analytical model (equation (2.6)) corresponding to the experimental data in Fig. 3(a).

For comparison with the experimental data, the analytical model is computed using a plate thickness $L$ of $0.55 \mathrm{~mm}$ and a wire depth $h$ of $0.4125 \mathrm{~mm}$ (i. .e. $3 \times 0.1375 \mathrm{~mm}$, which is the thickness of a single lamina). A defect depth $d$ of $0.1375 \mathrm{~mm}$ (i. .e. the thickness of a single lamina) and a defect diameter $D$ of $10 \mathrm{~mm}$ were assumed. Furthermore, to allow for imperfections in the bonding between patch and host material during the manufacturing process, it was assumed that the defect opening was $100 \mathrm{~mm}$ and that the defect filler was air. The thermal properties utilized for the laminated fiber-reinforced composite plate are given in Table 1.

The values in Table 1 were derived by fitting the results of the analytical model to those of the experimental data. In particular, the procedure consisted in two stages.

First The through-the-thickness thermal diffusivity $\alpha$ was derived by fitting the temperature contrast

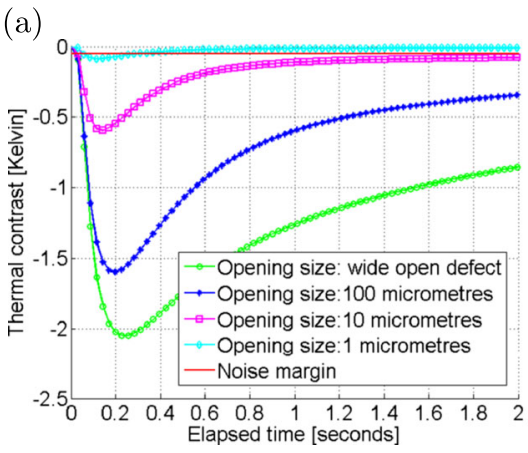

Defect depth $d=0.1375 \mathrm{~mm}$.

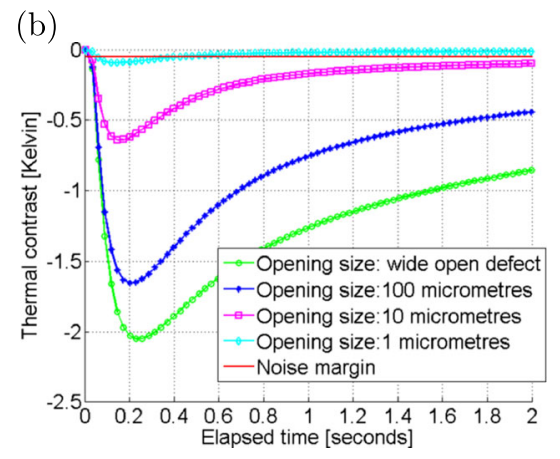

Defect depth $d=0.2750 \mathrm{~mm}$.

Fig. 4 Temperature contrast versus elapsed time curves computed using the analytical model (equation (2.6)) for four defect openings: wide open $(2.5 \mathrm{~cm}), 100 \mu \mathrm{m}, 10 \mu \mathrm{m}$ and $1 \mu \mathrm{m}$, and for two different defect depths: $0.1375 \mathrm{~mm}$ and $0.2750 \mathrm{~mm}$. For the curve in Fig. $4(\mathrm{~b})$ with the defect opening $100 \mu \mathrm{m}$ the minimum temperature contrast and time are $T_{c_{\min }}=-1.66 \mathrm{~K}$ and $t_{\min }=0.21 \mathrm{~s}$, which are respectively $\approx 3 \%$ and $\approx 10 \%$ greater than for the same curve in Fig. 4(a) 


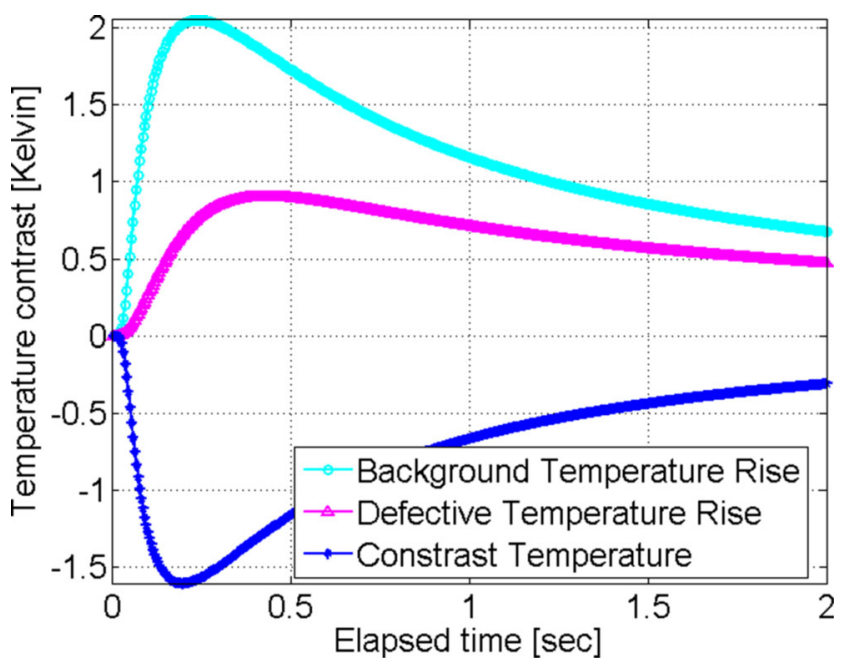

Fig. 5 Temperature contrast versus elapsed time computed using the approximate expression (equation (2.10)) for the same parameters utilized in Fig. 3(b). In this case the minimum temperature contrast $T_{c_{\min }}=-1.61 \mathrm{~K}$ at time $t_{\min }=0.20 \mathrm{~s}$

obtained experimentally to the one computed using equation (2.6) for a value of $\Gamma=1$, which corresponds to a null contribution of the defective temperature rise.

Second The thermal anisotropy $A$ was derived by fitting the defective temperature rise obtained experimentally to the one computed from equation (2.5). The fitting was performed at a distance $\mathrm{D} / 2=2.5 \mathrm{~mm}$ from the edge of the defect, where the lateral diffusion was seen to be especially strong in the thermal images.

The non-defective and defective temperatures in Fig. 3(a) are constructed using, respectively, $m=0, \ldots, 10$ in equation (2.3) and $m=0, \ldots, 10$ and $n=0, \ldots, 10$ in equation (2.4). The temperature contrast computed by the analytical model agrees quite well the the experimental results in Fig. 3(a), with the model predicting a trough in the temperature contrast of $-1.60 \mathrm{~K}$ at $0.19 \mathrm{~s}$.

In Table 2 the results predicted by the analytical model (M) (equation (2.6)) and its approximate expression (AM) (equation (2.10)) for the minimum contrast time $t_{\min }$ are compared against the experimental ones for $10 \mathrm{~ms}, 20 \mathrm{~ms}$, $30 \mathrm{~ms}, 40 \mathrm{~ms}$ and $50 \mathrm{~ms}$ heating periods.

Figure 4(a) shows the temperature contrast versus elapsed time curves computed using the analytical model (equation (2.6)) for four defect openings: wide open $(2.5 \mathrm{~cm}), 100 \mu \mathrm{m}, 10 \mu \mathrm{m}$ and $1 \mu \mathrm{m}$, holding fixed all other parameters. These curves are compared with a noise margin of $0.05 \mathrm{~K}$ that is taken as being the minimum temperature contrast necessary to produce a useful thermal image of a defect. The results change significantly depending on the defect opening being considered, as was also found in [10], with the minimum contrast temperature and time in these curves increasing with defect depth.

Figure 4(b) shows the temperature contrast versus elapsed time curves for same parameters in Fig. 4(a) but doubling the defect depth to $0.2750 \mathrm{~mm}$. In this case for the defect opening $100 \mathrm{~mm}$ the minimum temperature contrast is $T_{c_{\text {min }}}=-1.66 \mathrm{~K}$ at time $t_{\text {min }}=0.21 \mathrm{~s}$ which are respectively $\approx 3 \%$ and $\approx 10 \%$ greater than for the same curve in Fig. 4(a), which gives an indication of the parameter sensitivity of the minimum contrast temperature and position for changes in the defect depth. The sensitivity of the temperature contrast and position to changes in defect depth using the approximate expression (equation (2.10)) will be analyzed in Fig. 6.

In Fig. 4 defects with all of the four openings exceed the noise margin, indicating SMArt thermograhy to be suitable as a TNDE technique for the inspection, but the sensitivity of the contrast to defect opening is evident, with the temperature contrast curve for the $1 \mathrm{~mm}$ opening size just above the noise margin.

Figure 5 shows the temperature contrast versus elapsed time computed using the approximate expression (equation (2.10)) for the same parameters utilized in Fig. 3(b).
Fig. 6 Sensitivity of minimum contrast temperature and time versus defect depth using the approximate expression (equation (2.10)) for the two defect depths in Fig. 4. Note how minimum contrast temperature and time are not very sensitive to variations of the defect depth using this approximate expression, as a $\approx 50 \%$ variation in defect depth causes only a couple of percentage points variation in these parameters (a)

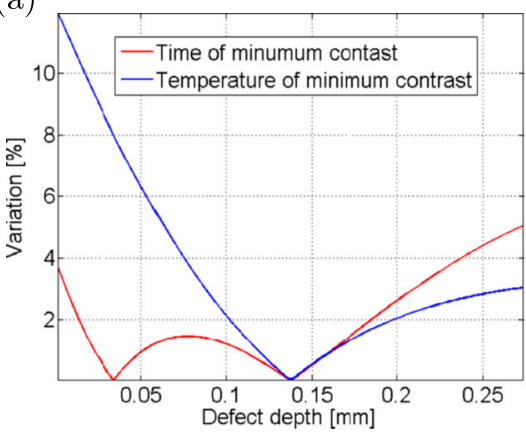

Defect depth $d=0.1375 \mathrm{~mm}$.

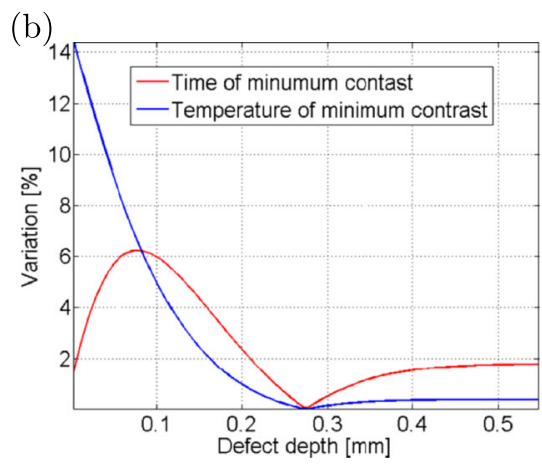

Defect depth $d=0.2750 \mathrm{~mm}$. 
In this case the minimum temperature contrast is $T_{c_{\min }}=$ $-1.61 \mathrm{~K}$ at time $t_{\text {min }}=0.20 \mathrm{~s}$. Plugging these values into the expression of $d_{1}$ given by equation (2.12) gives the correct answer for the defect depth $1.375 \times 10^{-4} \mathrm{~m}$.

Figure 6 shows the sensitivity of minimum contrast temperature and time to variations in the defect depth using the approximate expression (equation (2.10)) for the two defect depths in Fig. 4. Minimum contrast temperature and time were not found to be very sensitive to variations of the defect depth using this approximate expression, as a $\approx 50 \%$ variation in defect depth causes only a couple of percentage points variation in these parameters. For this reason, small errors in the minimum contrast temperature and time might produce large errors in the defect depth when computed analytically using the expression (equation (2.12)).

Figure 7 shows the results predicted for the defect depth $d$ by $d_{1}$ in equation (2.12) from the experimental data for the $10 \mathrm{~ms}, 20 \mathrm{~ms}, 30 \mathrm{~ms}$ and $40 \mathrm{~ms}$ heating periods. These graphs are plotted against the width of the plate, as this is the dimension along which the SMA wire is running through the plate (i. e. the abscissas in Fig. 2). Contrast (Fig. 7(a)-(d)) to Fig. 2(b) to have a visual comparison of the defect size and location. The differences between the maximum predicted value in Fig. 7(a)-(d), compared to the assumed value $\approx 0.1375 \mathrm{~mm}$, range between $1-7 \%$.
The defect depth was evaluated using the following procedure.

1. Temperature contrast curves were generated from the experimental data, using the same non-defective temperature rise computed at a small area located on top of the SMA wire, and were smoothed locally using simple moving averaging.

2. The defect depth was then evaluated at each location along the wire employing the expression for $d_{1}$ in equation (2.12) where the minimum temperature $T_{c_{\text {min }}}$ and time $t_{\text {min }}$ are computed from the temperature contrast curve of the experimental data.

There will be small positive or negative values of contrast temperature for all non-defective regions compared to the reference one, because of local differences in the heat conduction phenomena and noise in the thermographic system. These can be safely discarded in three ways:

(a) by setting to zero the defect depth when the contrast temperature is found to be positive;

(b) by setting to zero the defect depth when the contrast temperature is found to be negative, but is far away from the location of the defect (which is assumed to have been previously estimated from the thermal images), and
Fig. 7 Results predicted for the defect depth $d$ by $d_{1}$ in equation (2.12) from the experimental data for the $10 \mathrm{~ms}, 20 \mathrm{~ms}, 30 \mathrm{~ms}$ and $40 \mathrm{~ms}$ heating periods. These graphs are plotted against the width of the plate, as this is the dimension along which the SMA wire is running through the plate (i. e. the abscissas in Fig. 2). Contrast (Fig. 7(a)-(d)) to Fig. 2(b) to have a visual comparison of the defect size and location. The differences between the maximum predicted value in Figs. 7(a)-(d), compared to the assumed value $\approx 0.1375 \mathrm{~mm}$, range between 1-7\%
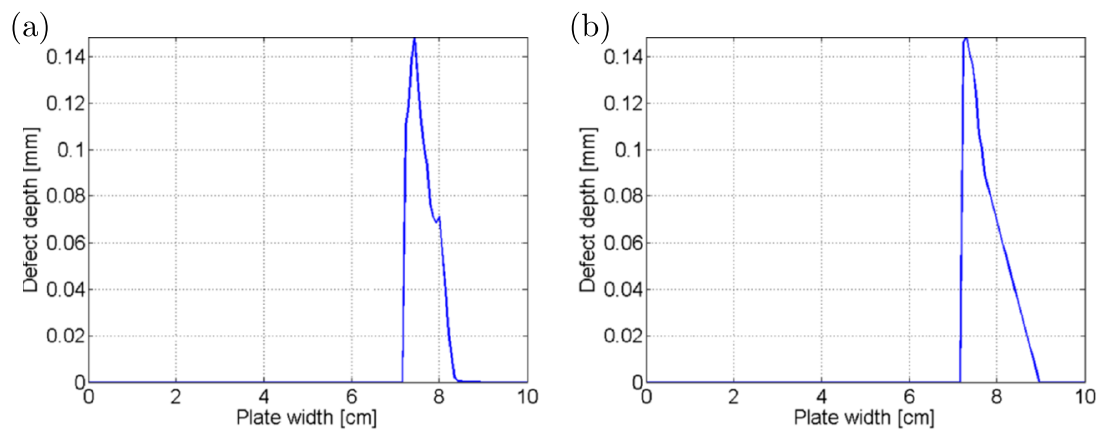

$10 \mathrm{~ms}$ heating period.

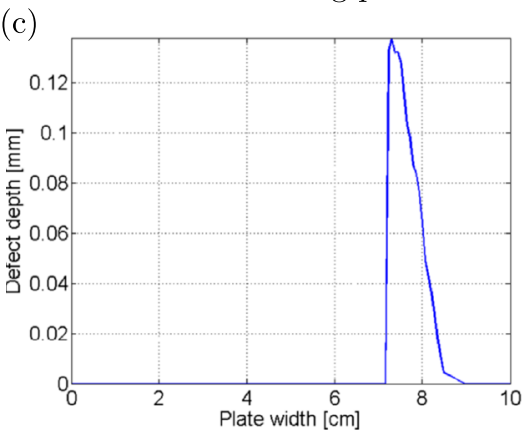

$30 \mathrm{~ms}$ heating period. (d)

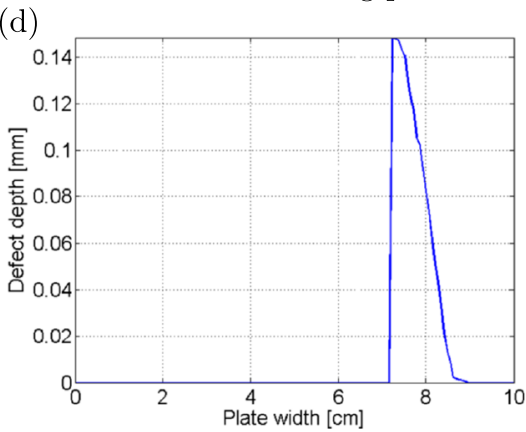

$40 \mathrm{~ms}$ heating period. 
(c) by removing from the results any complex or negative values of defect depth.

In fact, by construction, expression (equation (2.12)) will produce a real positive, physically meaningful result for the defect depth only for values of contrast temperature and time that fit closely the curve in Fig. 5.

3. Finally, previously discarded results for the defect depth were reconstructed using inpainting techniques, which aim at filling-in holes in digital data by propagating surrounding data (e.g. [31]), the only condition being that the defect depth on the boundaries of the plate should be zero.

In Figs. 5, 6 and 7 the defect diameter $D$ is assumed to have been estimated precisely from the thermal images. In real applications a component would carry a grid of SMA wires, at known distances and depths, that can be used to estimate the extent of the damage, hence the value of the diameter to be used in the expression for $d_{1}$ in equation (2.12). A methodology for estimating the size of the internal damage based on the a priori knowledge of the inter-wire distance and length is discussed in [20].

Despite good agreement with the experimental data (errors ranging between 1-7\%), some limitation must be highlighted. The analytical expression for the defect depth assumes known material and defect thermal properties, geometry of the sample and defect diameter and opening. While the defect diameter can be estimated from the thermal images, it is not possible to estimate the defect opening. All that is feasible is to evaluate the defect depths for a range of defect openings for a specific application. Furthermore, minimum contrast temperature and time using the approximate expression were not found to be very sensitive to large variations of the defect depth, but highly dependent on defect opening.

\section{Conclusions}

This paper developed an analytical model to derive an approximate expression for evaluating the defect depth for pulsed thermography performed using embedded SMA wires in laminated composites. The model was compared against experimental data and showed a good agreement in predicting the general form of the contrast curves for images of defects and for predicting the time and magnitude of the peak in contrast. In addition, the results for the defect depth computed showed vey good agreement with the experimental data (errors ranging between 1-7\%). This paper showed that SMart thermography and the developed analytical model could be used as a quantitative nondestructive tool where defect size and depth could be estimated with good accuracy.

Open Access This article is distributed under the terms of the Creative Commons Attribution 4.0 International License (http:// creativecommons.org/licenses/by/4.0/), which permits unrestricted use, distribution, and reproduction in any medium, provided you give appropriate credit to the original author(s) and the source, provide a link to the Creative Commons license, and indicate if changes were made.

\section{References}

1. Milne J, Reynolds W (1985) The non-destructive evaluation of composites and other materials by thermal pulse video thermography. In: 1984 Cambridge Symposium, International Society for Optics and Photonics, pp 119-122

2. Shepard SM (2001) Advances in pulsed thermography

3. Maldague X (1993). In: 1 (ed) Nondestructive Evaluation of Materials by Infrared Thermography. Springer, London

4. Chai H, Babcock CD, Knauss WG (1981) One dimensional modelling of failure in laminated plates by delamination buckling. Int J Solids Struct 17(11):1069-1083

5. Bolotin VV (1996) Delaminations in composite structures: its origin, buckling, growth and stability. Compos Part B: Eng 27(2):129-145

6. Polimeno U, Meo M (2009) Detecting barely visible impact damage detection on aircraft composites structures. Compos Struct 91(4):398-402

7. Lau S, Almond D, Milne J (1991) A quantitative analysis of pulsed video thermography. NDT E Int 24(4):195-202

8. Almond DP, Lau S (1993) Edge effects and a method of defect sizing for transient thermography. Appl phys Lett 62(25):33693371

9. Almond DP, Lau S (1994) Defect sizing by transient thermography. i. an analytical treatment. J Phys D Appl Phys 27(5): 1063

10. Saintey M, Almond DP (1995) Defect sizing by transient thermography. ii. a numerical treatment. J Phys D Appl Phys 28(12):2539

11. Ludwig N, Teruzzi P (2002) Heat losses and 3d diffusion phenomena for defect sizing procedures in video pulse thermography. Infr Phys Technol 43(3-5):297-301

12. Sun J (2006) Analysis of pulsed thermography methods for defect depth prediction. J Heat Transf 128(4):329-338

13. Zeng Z, Li C, Tao N, Feng L, Zhang C (2012) Depth prediction of non-air interface defect using pulsed thermography. NDT E Int 48:39-45

14. Roth D, Bodis J, Bishop C (1997) Thermographic imaging for high-temperature composite materialsa defect detection study. J Res Nondestruct Eval 9(3):147-169

15. Almond DP, Pickering SG (2012) An analytical study of the pulsed thermography defect detection limit. J Appl Phys 111(9):093510

16. Almond DP, Pickering SG (2014) Analysis of the defect detection capabilities of pulse stimulated thermographic nde techniques. AIP Conf Proc 1581(1):1617-1623 
17. Lau S, Almond D, Patel P (1991) Transient thermal wave techniques for the evaluation of surface coatings. J Phys D Appl Phys 24(3):428

18. Almond DP, Patel P (1996) Photothermal science and techniques, 1st Edi tion, Vol. 10 of Chapman \& Hall Series in Accounting and Finance (Book 10). Springer, London

19. Bennett Jr C, Patty R (1982) Thermal wave interferometry: a potential application of the photoacoustic effect. Appl Opt 21(1):49-54

20. Pinto F, Ciampa F, Meo M, Polimeno U (2012) Multifunctional smart composite material for in situ $\mathrm{ndt} / \mathrm{shm}$ and de-icing. Smart Mater Struct 21(10): 105010

21. Rogers CA (1990) An introduction to intelligent material systems and structures. Intelligent structures:3-41

22. Thomas JP, Qidwai MA (2004) Mechanical design and performance of composite multifunctional materials. Acta Mater 52(8):2155-2164

23. Angioni SL, Meo M, Foreman A (2011) Impact damage resistance and damage suppression properties of shape memory alloys in hybrid composites-a review. Smart Mater Struct 20(1): 013001
24. Nagai H, Oishi R (2006) Shape memory alloys as strain sensors in composites. Smart Mater Struct 15(2):493

25. Xu Y, Otsuka K, Nagai H, Yoshida H, Asai M, Kishi T (2003) A sma/cfrp hybrid composite with damage suppression effect at ambient temperature. Scr Mater 49(6):587-593

26. Pinto F, Maroun F, Meo M (2014) Material enabled thermography. \{NDT\} E Int 67(0):1-9

27. Carlslaw HS, Jaeger JC (1959) Conduction of Heat in Solids, 2nd edn. Oxford University Press

28. Patel P, Almond D, Reiter H (1987) Thermal-wave detection and characterisation of sub-surface defects. Appl Phys B 43(1):9-15

29. MATLAB, version 8.0.0.783 (R2012b), The MathWorks Inc., Natick, Massachusetts, 2012. www.mathworks.com

30. Ciampa F, Pickering S, Scarselli G, Meo M (2014) Nonlinear damage detection in composite structures using bispectral analysis. In: SPIE Smart Structures and Materials Nondestructive Evaluation and Health Monitoring, International Society for Optics and Photonics, pp 906402-906402

31. Garcia D (2010) Robust smoothing of gridded data in one and higher dimensions with missing values. Comput Stat Data Anal 54(4):1167-1178 